\title{
The study of stress-strain state of stabilized layered soil foundations
}

\author{
Mikhail V. Sokolov ${ }^{1}$, Sergey M. Prostov ${ }^{1 *}$, and Viktor S. Zykov ${ }^{2}$ \\ ${ }^{1}$ T.F. Gorbachev Kuzbass State Technical University, Vesennyaya St. 28, Kemerovo city, Russia \\ ${ }^{2}$ VNIMI, Sovetckiy St. 63a, Kemerovo city, Russia
}

\begin{abstract}
Herein presented are the results of modeling and analysis of stress-strain state of layered inhomogeneous foundation soil when it is stabilised by injection to different depths. Produced qualitative and quantitative analysis of the components of the field of isolines of stresses, strains, stress concentration and the difference between the strain at the boundary of different elastic horizontal layers. Recommendations are given for the location of stabilised zones in relation to the border of different elastic layers. In particular, it found that stabilization of soil within the weak layer is inappropriate, since it practically provides no increase in the stability of the soil foundation, and when performing stabilisation of soil foundations, it is recommended to place the lower border of the stabilisation zone below the border of a stronger layer, at this the distribution of stresses and strains occurs more evenly, and load-bearing capacity of this layer is used to the maximum.
\end{abstract}

\section{Introduction}

In construction of mining buildings, civil and industrial buildings there is a frequent situation when soil foundations are made of two or more soils with different physical and mechanical properties while weak strata of the massif are located on top of more solid soil [1, 2]. The inhomogeneity of rock strata properties, in particular, its layered structure, considerably affects the distribution of stresses and strains [3-6]. The study of geomechanical aspects of the problem will improve the accuracy of prediction of soil foundations stability including foundations fixed by injection methods, as well as reduce the risks of technological accidents at the facilities of the mining and construction industries.

When solving such problems, the method of numerical simulation becomes increasingly common, its main provisions are described in several papers [7-10]. The concepts of the method are implemented in the framework of "Alterra" software for geotechnical calculations made by company "InzhStroyProekt". The basic principles of modeling and analysis of computer calculations are set out in the work [11].

The base model for the formation of a database and further analysis (Fig. 1) is implemented for the two-step strip reinforced concrete foundation loaded with a vertical concentrated force $P=154.7 \mathrm{kN}$, the height $d_{f}=3.0 \mathrm{~m}$ and the following mechanical parameters: modulus of deformation $E_{f}=2000 \mathrm{MPa}$; Poisson's ratio $v_{f}=0.15$; density $\rho_{f}=2500 \mathrm{~kg} / \mathrm{m}^{3}$.

\footnotetext{
* Corresponding author: psm.kem@mail.ru
} 
Soil massif consists of the upper (layer \#1) and lower (layer \#2) layers with specified physical and mechanical properties in accordance with examples of calculation [12-14] and SP 23.13330.2011 (Table 1), layers thickness $H_{1}=H_{2}=10.5 \mathrm{~m}$, the width of the model $B_{m}=31.2 \mathrm{~m}$.

Formation of the database for the analysis was carried out by setting the increment of physical and mechanical properties of the massif soils in the range of relations $E_{2} / E_{1}=1 \ldots 5$ (Table 1 ) with various schemes of ground stabilisation by injection according to the recommendations adopted in the works $[15,16]$ :

- at location of zones of stabilisation within the boundaries of the weak layer (Fig.1,a);

- when stabilisation zones rest upon the border of layers (Fig.1,b);

- when the stabilisation zones are jammed in the strong layer (Fig.1,c).

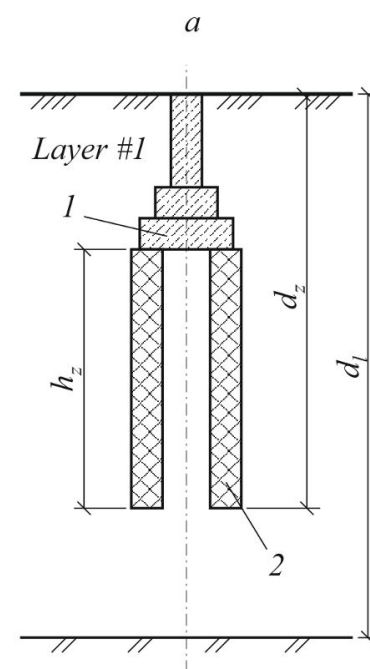

Layer \#2

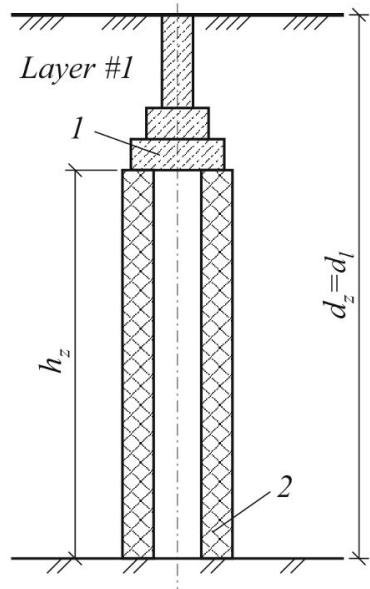

Layer \#2

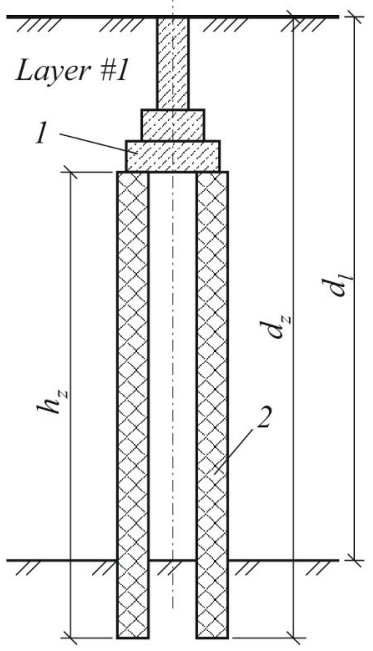

Layer \#2

Fig. 1. Schemes of an arrangement of zones of stabilisation within the borders of the weak layer $(a)$, when rested upon $(b)$ and jammed in the strong layer $(c)$ : 1 - foundation; 2 - stabilisation zone

Table 1. Physical and mechanical properties of soils

\begin{tabular}{|c|c|c|c|c|c|c|}
\hline \multirow{3}{*}{ Properties } & \multirow{3}{*}{ Layer \#1 } & \multicolumn{5}{|c|}{ Layer \#2 } \\
\hline & & \multicolumn{5}{|c|}{ relationship of $E_{2} / E_{I}$} \\
\hline & & 1 & 2 & 3 & 4 & 5 \\
\hline Modulus of deformation $E_{i}, \mathrm{MPa}$ & 5 & 5 & 10 & 15 & 20 & 25 \\
\hline Poisson's ratio $v_{i}$ & 0.36 & 0.36 & 0.35 & 0.34 & 0.33 & 0.32 \\
\hline Average density $\rho_{i}, \mathrm{~kg} / \mathrm{m}^{3}$ & 1750 & 1750 & 1800 & 1850 & 1900 & 1950 \\
\hline Angle of internal friction $\varphi_{i}$, degrees & 17 & 17 & 18 & 19 & 20 & 21 \\
\hline Adhesion $C_{i}, \mathrm{kPa}$ & 5 & 5 & 10 & 20 & 30 & 40 \\
\hline
\end{tabular}

\section{Results and discussion}

Part of the results of simulation in the form of isolines fields of vertical stress $\sigma_{z}$ is shown in Fig. 2.

Vertical stresses $\sigma_{z}$ in the natural (non-bound) layered soil massif (Fig.2,a) are distributed symmetrically and have the area of concentration under the foundation, as well as characteristic changes on the border of layers. When forming the stabilization zones according 
to the schemes (Fig. 1), additional areas of stress concentration are formed under the bases of these zones, as well as along their lateral surfaces.

In analyzing the character of the distribution of vertical stress $\sigma_{z}$ from the depth the massif along the axis of symmetry of the foundation $z$ (fig. 3) it was established that the formation of the field of vertical stress concentration is observed at $z=7.5 \ldots 12.5 \mathrm{~m}$ depending on the location of the stabilisation zones and relations $E_{2} / E_{1}$. In particular, the greatest effect from stabilisation is achieved at scheme \#3 (Fig. 3,d), because in this case in the stabilised massif on the border of layers there are no surges of stress $\sigma_{z}$ due to their increase in the weak layer in the range $z=7.0 \ldots 10.0 \mathrm{~m}$, at the same time, the major part of the pressure is redistributed onto the strong layer.
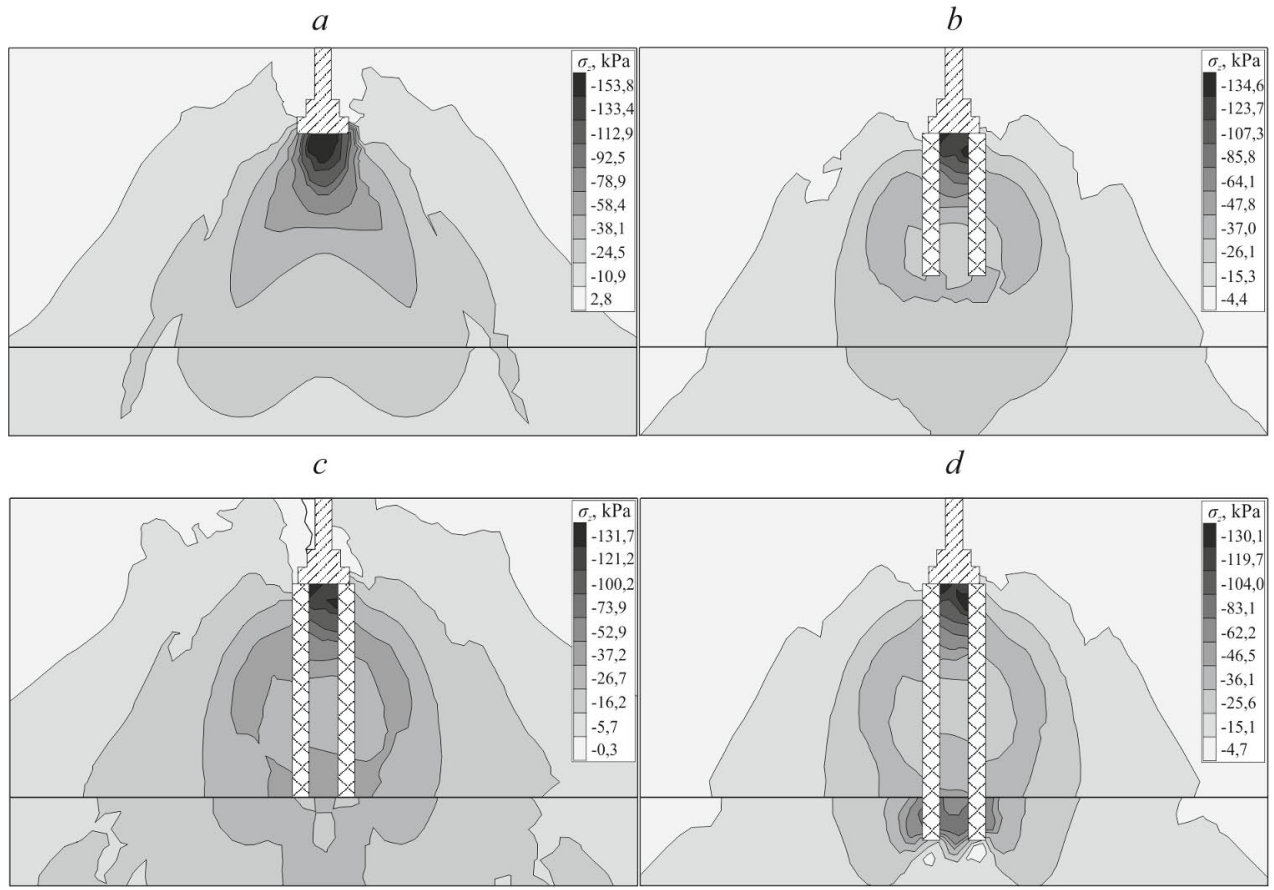

Fig. 2. Fields of isolines of vertical stress distribution in the natural soil foundation $(a)$, at the location of zones of stabilisation within the borders of the weak layer (b), when resting $(c)$ and jamming $(d)$ in the strong layer

Dependences of vertical stresses $\sigma_{z}$ on the depth of the model along the outer side surface of fixing zones $z_{s}$ are shown in Fig. 4.

The graphs show that at the location of stabilization zones in the weak layer (scheme \#1) the stresses decrease monotonically with increasing coordinate $z_{s}$ in the absence of local concentrations on the border of the layers. When the stabilised zone rests on this border (scheme \#2) and goes deeper in the strong layer (scheme \#3), the stress state of massif is fundamentally different: stresses $\sigma_{z}$ are more intensely redistributed to the lower layer.

For the numerical evaluation of the stress state of the soil massif, we introduce the integral criterion - stress concentration factor $k_{i}$, determined by the formula

$$
k_{i}=\frac{\sigma_{\max }}{\sigma_{\text {lost }}},
$$

where $\sigma_{\max }$ - the maximum stresses in the layer boundaries, $\mathrm{kPa} ; \sigma_{\text {lost }}-$ stress in the pristine part of the layer under consideration (at the border of the model), $\mathrm{kPa}$. 
a

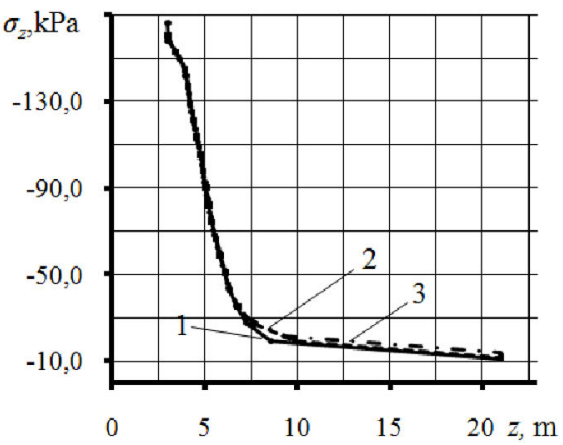

$c$

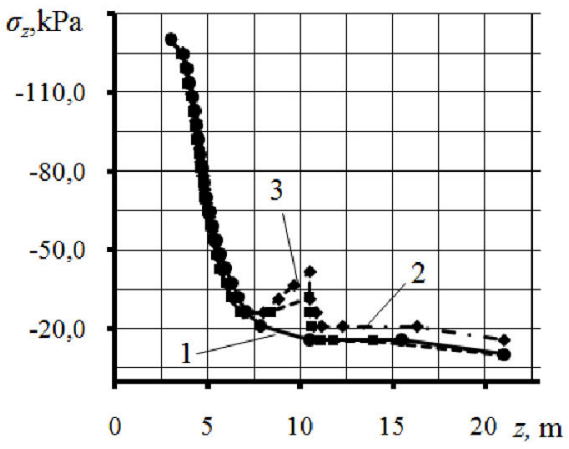

$b$

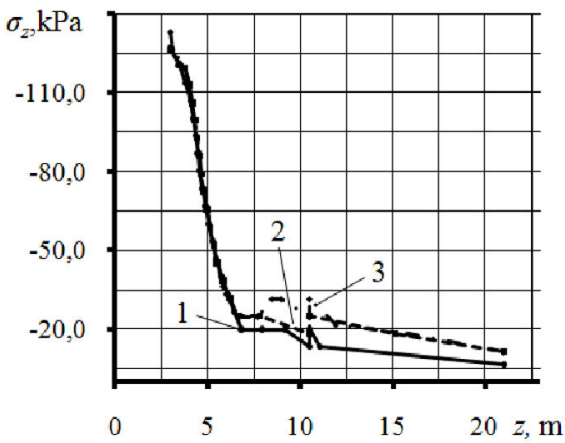

d

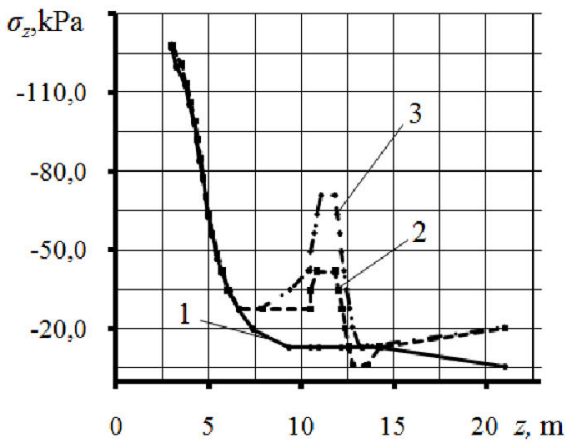

Fig. 3. The dependence of the stress $\sigma_{z}$ on the depth along the axis of the model $z$ in the natural $(a)$ and stabilised massif as per schemes \#1 (b), \#2 (c) and \#3 (d) and relation $E_{2} / E_{l}$ equal to: $1-$ $E_{2} / E_{1}=1 ; 2-E_{2} / E_{1}=3 ; 3-E_{2} / E_{1}=5$
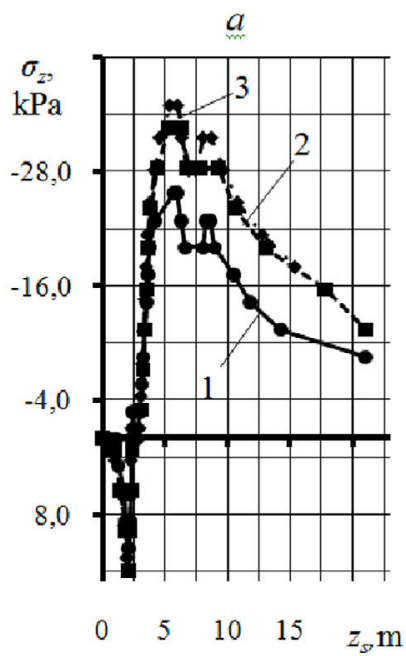

$b$

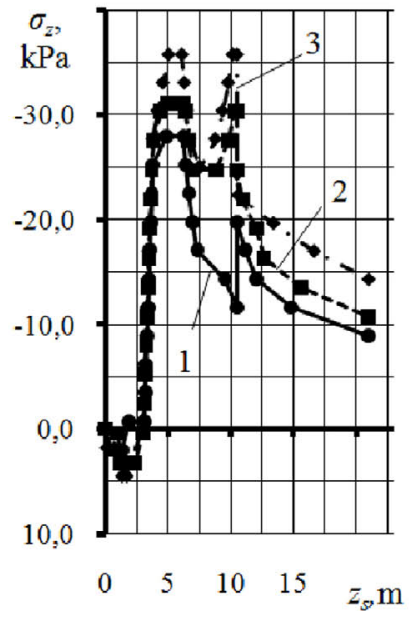

$c$

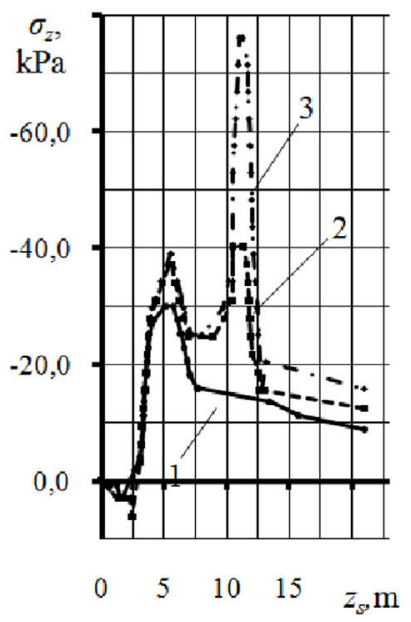

Fig. 4. Dependence of the stress $\sigma_{z}$ on the depth of model $z_{s}$ along the border of the stabilization zone when it is stabilized as per schemes \#1, \#2 and \#3, and relation of properties $E_{2} / E_{1}$, equal to: 1 $E_{2} / E_{1}=1 ; 2-E_{2} / E_{1}=3 ; 3-E_{2} / E_{1}=5$

The dependences of relation of the stress concentration factors $k_{1} / k_{2}$ in the weak and strong layers on relation $E_{2} / E_{1}$ are shown in Fig. 5. 


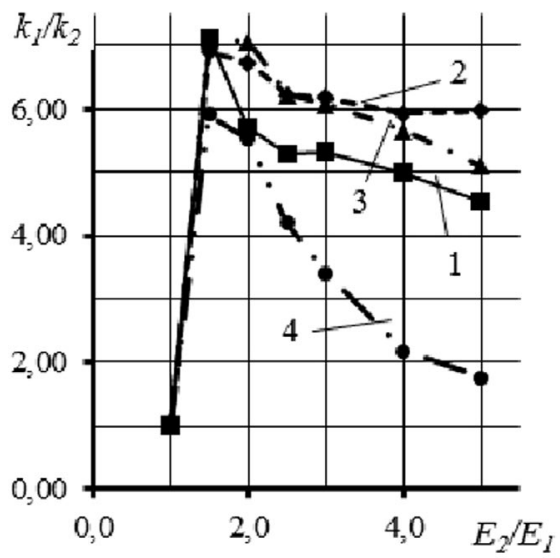

Fig. 5. Dependencies of relations $k_{1} / k_{2}$ on the relation $E_{2} / E_{1}: 1$ - natural massif; 2 - when stabilized as per scheme \#1;3 - scheme \#2; 4 - scheme \#3

According to the presented data, when relation $E_{2} / E_{1}$ increases in the range of $E_{2} / E_{1}=1.0 \ldots 2.5$, there occurs redistribution of stresses between the layers with formation of the area of maximum stresses. At stabilization as per the schemes \#1 and \#2, there is some smoothing of the graphs in the range of $E_{2} / E_{1}=2.0 \ldots 5.0$. The greatest effect is achieved at stabilization as per scheme \#3, where there is a sharp decline in $k_{1} / k_{2}$ to values close to 1 . Thus, the location of the base of the stabilisation zones within the boundaries of the strong layer contributes to a more favorable distribution of stresses in the layered soil massif.

The distribution pattern of vertical deformations $\varepsilon_{z}$ in the soil massif is shown by the fields of isolines shown in Fig. 6.
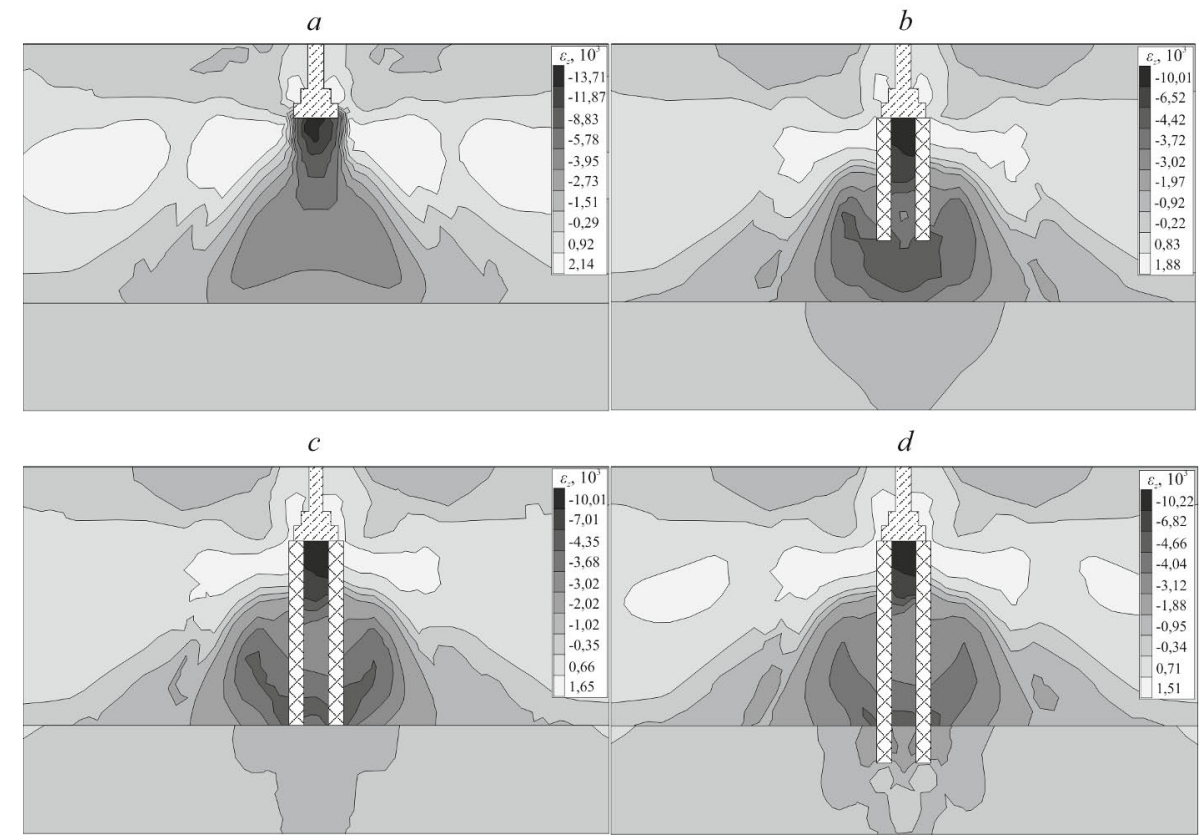

Fig. 6. Isoline fields of distribution of vertical deformations in the natural soil foundation $(a)$, at the location of stabilization zones within the borders of the weak layer $(b)$, at resting upon $(c)$ and jamming $(d)$ in the strong layer 
Distribution of deformations $\varepsilon_{z}$ occurs symmetrically, the areas of the maximum values stress are formed in specific parts of the massif: under the foundation at a depth of $z=3.0 \ldots 6.0 \mathrm{~m} 3.5-4.5 \mathrm{~m}$ wide, and when stabilized - in inter-areas space; under the lower ends of the stabilisation zones at an average width $5.5-6.5 \mathrm{~m}$, in some cases $3.2-4.0 \mathrm{~m}$.

Along the axis $z_{s}$ by the side face of the stabilisation zone (Fig. 7) there are significant lateral deformations of the stabilisation zones, since at the range of the depth $z_{s}=3.5-7.5 \mathrm{~m}$ (in some cases up to $10.5 \mathrm{~m}$ ) a change in the character of the deformation into compression is observed, as well as significant increase of $\varepsilon_{z}$.

Fig. 8 shows the dependence of the deformation difference at the border of layers $\Delta=\varepsilon_{z 2}-\varepsilon_{z l}$ on the relation $E_{2} / E_{l}$.

a

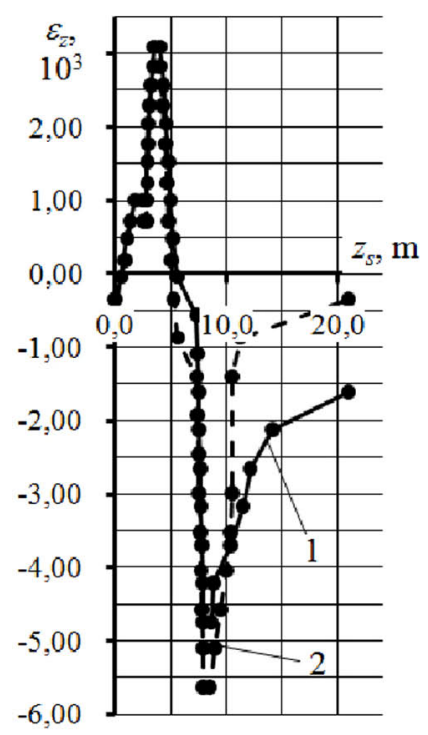

$b$

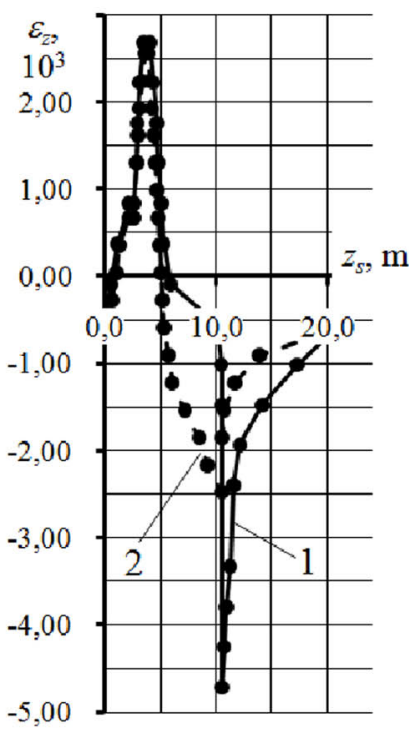

$c$

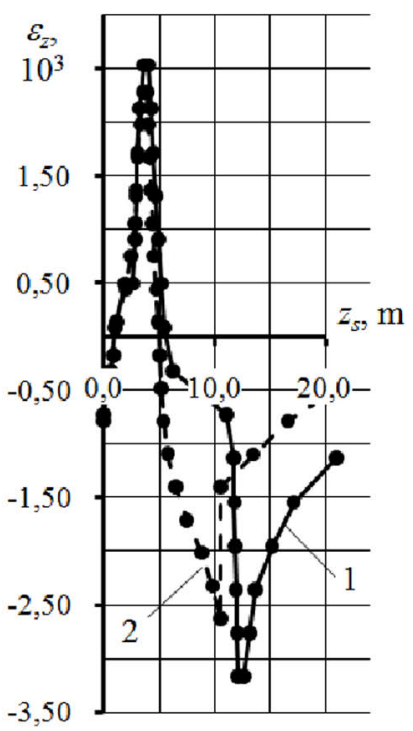

Fig. 7. Dependence of the vertical elastic deformations $\varepsilon_{z}$ on the depth of the model $z_{s}$ in the stabilised soil foundation according to schemes \#1 (a),\#2 (b) and \#3 (c), at relation $E_{2} / E_{1}$, equal to: 1 $E_{2} / E_{1}=1 ; 2-E_{2} / E_{1}=5$

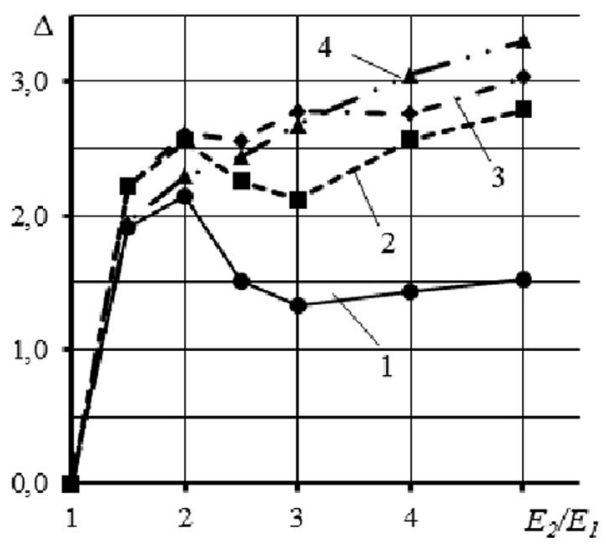

Fig. 8. Dependence of changes in the difference of the vertical deformation $\Delta$ on the border of layers on the relation $E_{2} / E_{1}: 1$ - natural massif; 2 - when stabilized as per schemes \#1;3 - schemes \#2; 4 scheme \#3 
The presented curves show that the highest values $\Delta$ are seen at stabilization as per scheme $\# 3$, at the same time the value $\Delta$ increases practically monotonously, without areas of local maximum values, in contrast to the other stabilization schemes. The lowest level of $\Delta$ is recorded in the natural massif, however, in the interval of relations $E_{2} / E_{1}=1.5 \ldots 2.5$ maximum values exceeding the value $\Delta=1.5$ at the ratio $E_{2} / E_{l}=5$ are observed.

\section{Conclusion}

As a result of the analysis of the stress-strain state of the layered stabilised soil massif, the following has been found:

- stabilization of soil within the weak layer is inappropriate, since it practically provides no increase in the stability of the soil foundation;

- when performing stabilization of soil foundations, it is recommended to place the lower border of the stabilization zone below the border of a stronger layer, at this the distribution of stresses and strains occurs more evenly, and load-bearing capacity of this layer is used to the maximum.

\section{References}

1. S. V. Usanov, V. I. Ruchkin, O. D. Zheltysheva, Journal of Mining Science, 51, 724 (2015)

2. O. B. Bocharov, V. Ya. Rudyak, A. V. Seryakov, Journal of Mining Science, 50, 235 (2014)

3. A. B. Ponomarev, E. N. Sychkina, Soil Mechanics and Foundation Engineering, 51, 111 (2014)

4. V. M. Seryakov, Journal of Mining Science, 45, 420 (2009)

5. Z. G. Ter-Martirosyan, A. Yu. Mirnyi. Soil Mechanics and Foundation Engineering. 50, 223 (2014)

6. V. I. Sheinin, E. P. Sarana, V. N. Soboleva, D. V. Gurevich, L. B. Katsnel'son, Soil Mechanics and Foundation Engineering, 53, 304 (2016)

7. V. E. Mirenkov, A. A. Krasnovsky. Journal of Mining Science, 49, 567 (2013)

8. S. G. Bezvolev. Soil Mechanics and Foundation Engineering, 45, 77 (2008)

9. V. V. Orekhov. Soil Mechanics and Foundation Engineering. 53, 1 (2016)

10. V.M. Ulitskii, M.A. Luchkin, Soil Mechanics and Foundation Engineering. 43, 197 (2006)

11. Sergei M. Prostov, Mikhail V. Sokolov and Andrey V. Pokatilov. International Journal of Applied Engineering Research. 10, 45297 (2015)

12. A. A. Tyrymov. Journal of Mining Science. 48, 812 (2012)

13. Mikhail Sokolov, Sergey Prostov. Advances in Engineering Research. 92, 206 (2016)

14. Sergei M. Prostov, Mikhail V. Sokolov. Taishan Academic Forum - Project on Mine Disaster Prevention and Control, 350 (2014)

15. M. N. Ibragimov. Soil Mechanics and Foundation Engineering. 46, 17 (2009)

16. S. M. Prostov, N. Yu. Nikulin. Journal of Mining Science. 51, 908 (2015) 